\title{
Frequency-coded chemical sensors
}

Francis Tsow, Erica Forzani and N.J. Tao*

Department of Electrical Engineering

Arizona State University, Tempe, Arizona 85287

\begin{abstract}
We have developed a new method to intelligently sample analytes and introduce the analytes to sensors. The method automatically adjusts sampling duration according to the sensors' response to the analytes, and converts the amplitude of the sensor output to a frequency output; giving us another opportunity to reduce noise in the signal. It also addresses some of the common sensor issues such as response time, saturation, chemical dynamic range and sensor protection; saving precious detection time, protecting sensors and enabling sensitive sensors built for low concentration detection to be used for high concentration detection as well. We have put together a system using a tuning fork chemical sensor as a sample sensor to demonstrate the feasibility and benefits of the new sensing technique.
\end{abstract}

*njtao@asu.edu 


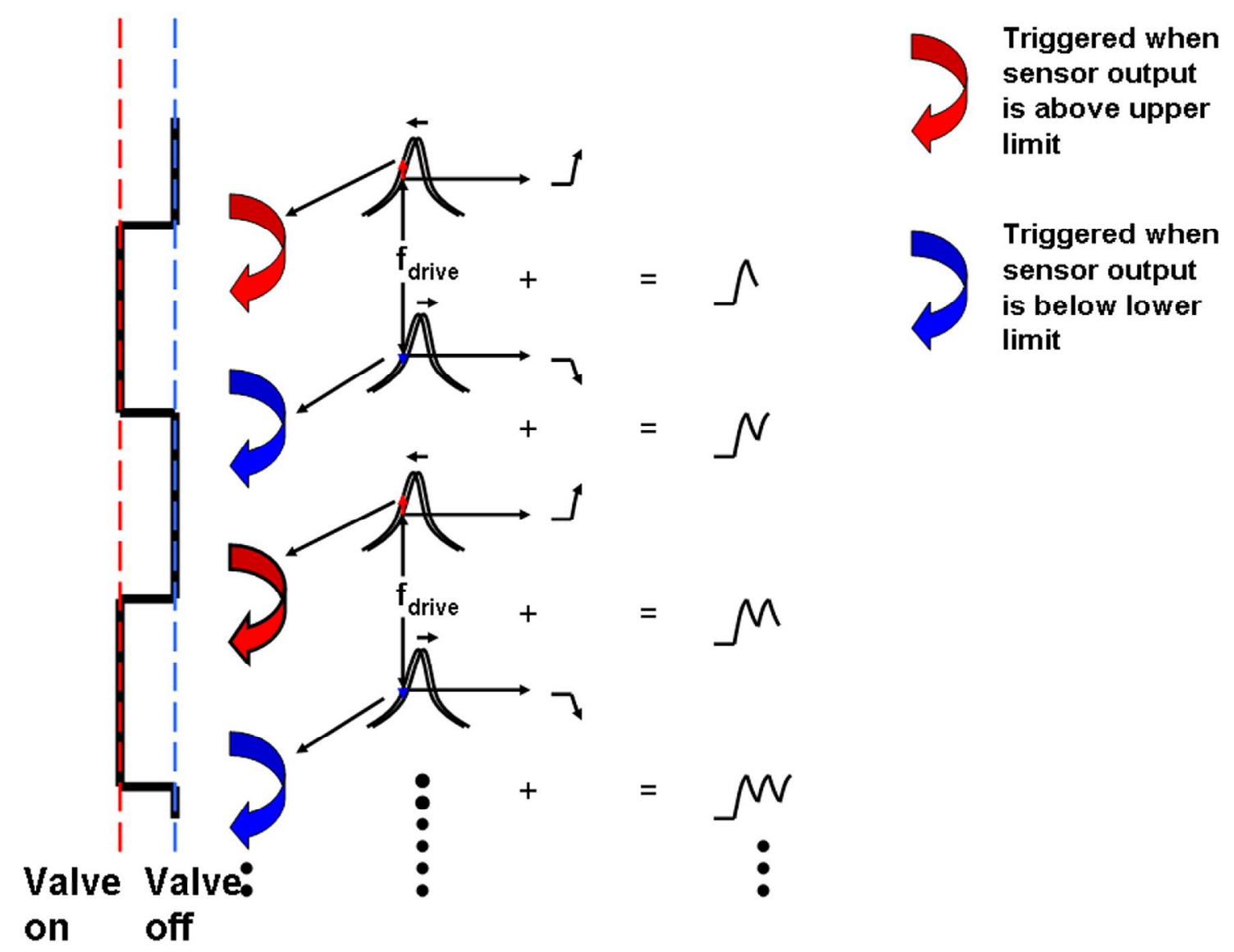

Figure S-1. Cartoon illustrating how the detection system works. The analyte vapor is pumped into the housing of the tuning fork sensor which triggers a change in the sensor signal. When the signal reaches a preset level $\left(\mathrm{V}_{\mathrm{hi}}\right)$, the microcontroller activates the valve which shuts off the analyte and at the same time allows purge gas $\left(\mathrm{N}_{2}\right.$ or air) to flow into the housing. As a result, the signal falls until it reaches another preset level, $\mathrm{V}_{\mathrm{lo}}$, at which the microcontroller re-exposes the sensor to analyte by activating the valve. This sequence of events, except the first ramp up, will then repeat itself until the experiment is stopped; resulting in a pseudo-oscillation. 


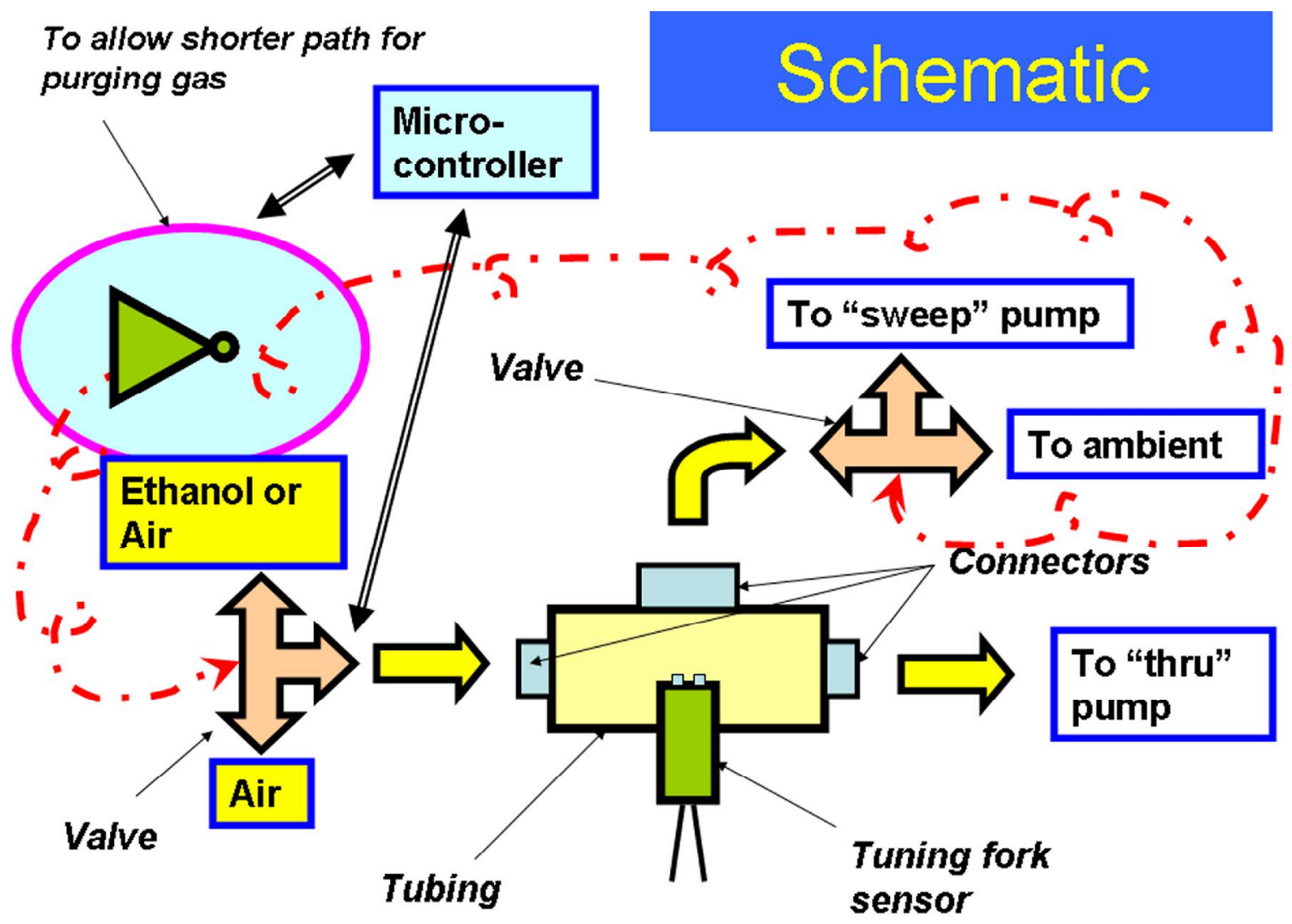

Figure S-2. Schematic of second sensing system employing a second 3-way valve and another high low rate pump (“sweep" pump) dedicated to help purging analyte. 


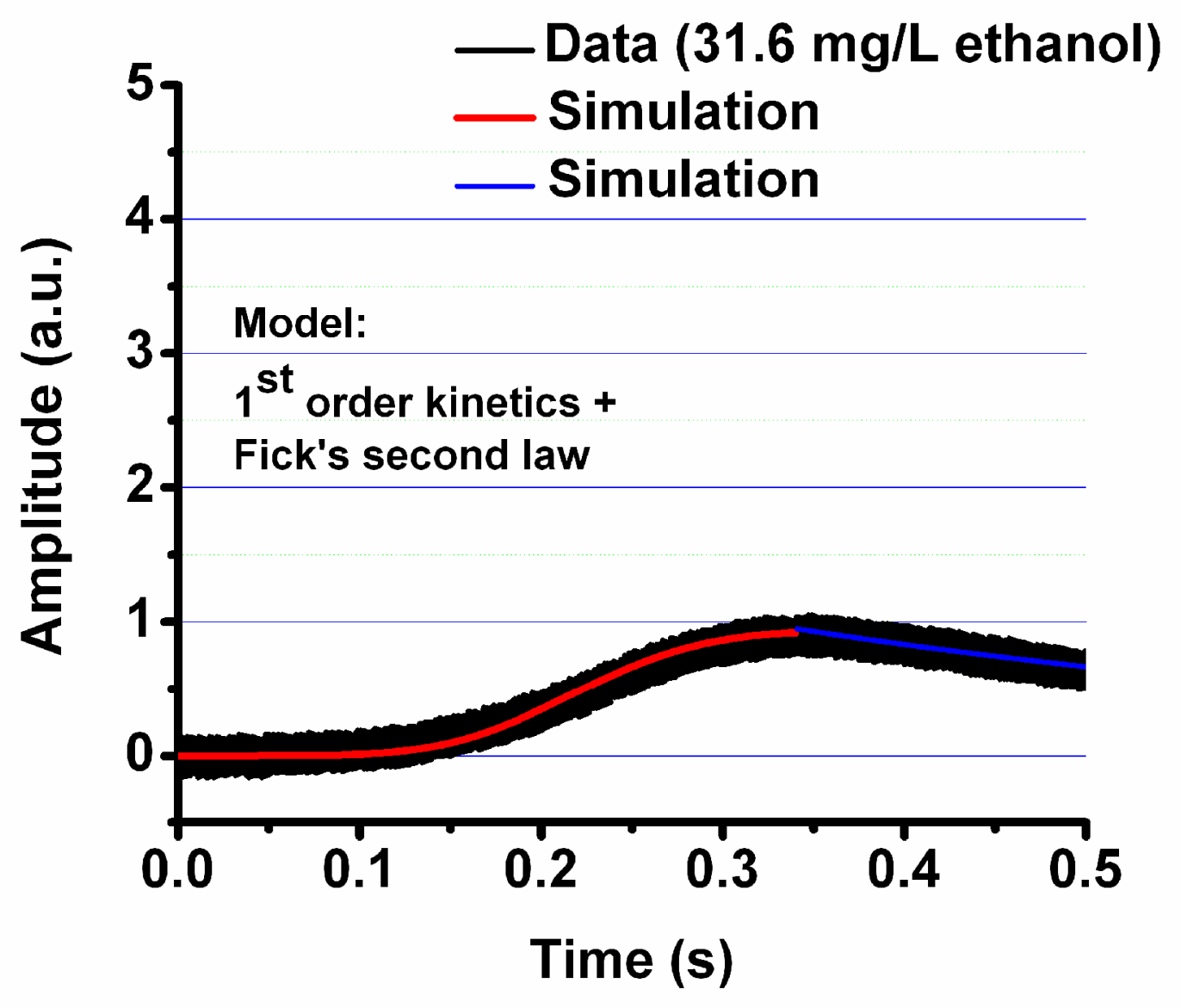

Figure S-3. Plots showing experimental data and simulation results. Data was obtained by passing $31.6 \mathrm{mg} / \mathrm{L}$ ethanol sample through frequency-coded sensor system. Data only shows one cycle of the system. First order kinetics and Fick's second law were used in the simulation. 